\title{
Effective Channel Hardening in an Indoor Multiband Scenario
}

\author{
Golsa Ghiaasi ${ }^{1}$ (1) · Jens Abraham ${ }^{1}$ - Egil Eide ${ }^{1} \cdot$ Torbjörn Ekman $^{1}$
}

Received: 14 February 2019 / Revised: 13 June 2019 / Accepted: 17 June 2019 / Published online: 2 July 2019

(c) The Author(s) 2019

\begin{abstract}
We evaluate channel hardening for a large scale antenna system by means of indoor channel measurements over four frequency bands, $1.472 \mathrm{GHz}, 2.6 \mathrm{GHz}, 3.82 \mathrm{GHz}$ and $4.16 \mathrm{GHz}$. NTNU's Reconfigurable Radio Network Platform has been used to record the channel estimates for 40 radio links to a 64 element array with wideband antennas in a rich scattering environment. We examine metrics for channel hardening, namely, the coherence bandwidth, the rms delay spread and the normalized effective subcarrier power, for the effective channel perceived by a single user after precoding and superposition in the downlink. We describe these metrics analytically and demonstrate them with measured data in order to characterize the rate of hardening of the effective channel as the number of antenna elements at the base station increases. The metrics allow for direct insight into the benefits of channel hardening with respect to radio system requirements.
\end{abstract}

Keywords Channel hardening · Massive MIMO $\cdot$ Delay spread

\section{Introduction}

Massive multiple input multiple output (MIMO) systems are envisioned as a key feature of the next generation of communication systems which provide large sum capacity as well as spectral and energy efficiency, while simultaneously serving multiple users. Some of the theoretical properties [2, 12, 18] have been empirically shown through recent measurement campaigns $[9,10,14]$. In these analyses, keeping the number of users 10 folds less than number of base station (BS) antennas is considered good practice.

The large scale antenna systems are being investigated as contenders for wireless sensor networks (WSNs) to offer mass connectivity with high reliability in $5 \mathrm{G}$ paradigm. In these systems, a BS equipped with a large number of antennas serves a very large number of sensor nodes such as ships, automobiles, trains, engines and robots, which are categorically referred to as user equipments (UEs). As many of these applications are safety critical, the robustness of the wireless communication links is vital. Channel hardening could be exploited, in order to establish reliable links between BS and UEs. By definition, the channel hardens when by increasing the number of BS antennas, the deviation of the gain of the

Golsa Ghiaasi

golsa.ghiaasi@ntnu.no

1 Department of Electronic Systems, Norwegian University of Science and Technology, Trondheim, Norway perceived channel at each UE decreases and the channel gain value becomes deterministic [11].

From a radio system perspective, the desirability of channel hardening at sensor nodes is twofold: The signals traveling from the BS to each single UE are precoded at the antenna elements and filtered by the physical channel and then will superpose and form what is referred to as effective channel. When the effective channel hardens, it is as if the propagation happened through a quasi-deterministic flat-fading equivalent channel by averaging out the small fading effects, as a result forming a reliable link from the BS to the UE. Considering channel hardening in the delay domain, complex equalization and estimation at the UE side can be avoided because the effective channel collapses to a single tap due to the delay dispersion being smaller then the delay tap resolution.

In $[8,15]$, the authors have looked at channel hardening for the subcarriers using maximum ratio combining (MRC) in the uplink (UL). The standard deviation is used to examine the dispersion of the channel. Alternatively, the work in [3] formulates the concept of an effective (equivalent) received channel at a single UE by using timereversal precoding. Here, the measured channels between the UE and the BS are used to examine temporal focusing by using the delay spread and the strongest tap power distribution. In [16] the measured channels between a 128 antenna BS and 36 UEs have been used to evaluate the root 
mean square (rms) delay spread of the effective combined channel for three common linear precoding schemes.

In this paper, we refer to the perceived channel at a single user in the downlink (DL) as an effective channel. This is the channel formed by precoding, propagation and superposition of signals from each BS antenna element. A calibration of the transmitter and receiver chains at the $\mathrm{BS}$ is necessary, such that the reciprocity assumption holds [7].

Channel hardening is considered from two points of view: firstly, as a property that causes the effective channel transfer function (CTF) between the UE and the BS to become more deterministic, secondly as a property to focus the received signal in the delay domain as the number of BS antennas increases. We illustrate these properties in the effective channel in order to determine how many antennas are sufficient to achieve a certain level of channel hardening. This will allow for the remaining BS antennas to be considered as contributors to achieve a multi-user system by using the remaining degrees of freedom to orthogonalize the effective channels.

We base our analysis on a channel dependent precoding which weights the signal at the antenna elements and relies on exploiting channel reciprocity in the DL to form a matched filter combination when observed by the UE. The aim of the precoding is to guarantee the best average signal to noise ratio (SNR) at the UE under the assumption that the channel state information (CSI) of the channel is perfectly known and no co-channel interference exists.

We formulate the normalized effective subcarrier power in order to examine the flatness of the effective channel at the UE. Additionally, the coherence bandwidth as well as the effective delay spread are analyzed. All three metrics have been characterized with measurement data at 4 frequency bands: $1.472 \mathrm{GHz}, 2.6 \mathrm{GHz}, 3.82 \mathrm{GHz}$ and $4.16 \mathrm{GHz}$. These constitute the commonly considered frequency range for $5 \mathrm{G}$ and WSN systems and highlight potential frequency dependencies.

This manuscript is organized as follows: in Sect. 2 the measurement campaign which was carried out in an indoor area with industrial profile forming a quasi-static scenario is reviewed. The acquired data corresponds to 40 spatial sample points characterizing UL channels to a 64 element array in the above-mentioned frequency bands. The details of this campaign are reported in [6]. In Sect. 3, the concept of the effective channel is introduced and the measured channel data is used to analytically form the effective channels. The metrics of hardening are described and evaluated in Sect. 4. We show that coherence bandwidth ceases to be a practical measure for effective channel evaluation. Lastly, the conclusions are presented in Sect. 5.

\section{Measurement Description}

The investigation reported in this paper is based on the measured data acquired during a campaign carried out at the Norwegian University of Technology and Science (NTNU) in December 2017 using the Reconfigurable Radio Network Platform (ReRaNP). The details of the UEs and the BS including channel estimate acquisition are described in [6].

\subsection{Measurement Setup}

For our BS, we used two modules of the NTNU massive MIMO testbed with 32 National Instruments (NI) USRP2943R devices. Two RF chains exist in each Universal Software Radio Peripheral (USRP) unit, as a result we have 64 radio chains at the BS. These units are controlled by one CPU which is running the NI LabVIEW Communications MIMO Application Framework. They form a time division duplex (TDD) system with a Long Term Evolution (LTE)like physical layer with $20 \mathrm{MHz}$ operational bandwidth [1].

To implement the UEs, the radio chains of four NI USRP2953R units were controled through NI LabVIEW Communications MIMO Application Framework in Mobile configuration. The key parameters of the system are summarized in Table 1.

The BS antenna array and UE are equipped with wideband Log-Periodic Dipole Arrays (LPDAs) covering the frequency band between 1.3 and $6.0 \mathrm{GHz}$. The linearly polarized LPDA element has been designed to provide a half power beamwidth of approximately $110^{\circ}$ in the H-plane and $70^{\circ}$ in the E-plane giving a directive gain of $6 \mathrm{dBi}$ when used as a single element. Each UE is equipped with one LPDA antenna element in vertical polarization, whilst the BS is equipped with 4 subarrays each containing 32 LPDA elements in an equally spaced $4 \times 8$ rectangular configuration as illustrated in Fig. 1. The antennas in the arrays are mounted

Table 1 Massive MIMO testbed parameters

\begin{tabular}{ll}
\hline Parameter & Value \\
\hline \# of BS antennas & $64 \mathrm{used}$ \\
\# of UEs & 8 \\
Center frequency & $1.2-6 \mathrm{GHz}$ \\
Bandwidth of operation & $20 \mathrm{MHz}$ \\
Baseband sampling rate & $30.72 \mathrm{MS} / \mathrm{s}$ \\
Subcarrier spacing & $15 \mathrm{kHz}$ \\
\# of subcarriers & 1200 \\
FFT size & 2048 \\
Frame duration & $10 \mathrm{~ms}$ \\
Subframe duration & $1 \mathrm{~ms}$ \\
Slot duration & $0.5 \mathrm{~ms}$ \\
TDD periodicity & $1 \mathrm{~ms}$ \\
\hline
\end{tabular}




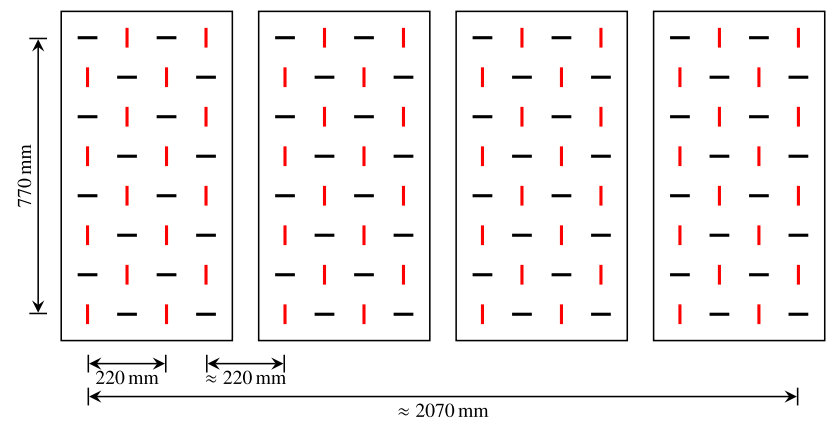

Fig. 1 Frontal view of the antenna element configuration. Only vertically oriented elements were used for the reported measurement campaign

with an element spacing of $110 \mathrm{~mm}$ on a common ground plane. As shown in Fig. 1 the antenna elements have interleaved polarization such that each element has a neighbor with orthogonal polarization. This reduces the mutual coupling effects between the elements to a minimum, hence the effect on the input impedance and radiation pattern for the elements are minimized. 64 vertically polarized elements were terminated at the BS whilst the other 64 horizontally polarized elements were left open. The configuration is depicted in Fig. 1.

\subsection{Measurement Scenario}

The measurement campaign was carried out in an indoor space with industrial profile in presence of glass, stone and metal reflecting surfaces. As depicted in Fig. 2 the BS was set up at the balcony at the end of the long hall while the cart containing the 8 UEs was placed on a wheeled cart at around 15 meter distance from the BS. The antennas of the UEs are positioned in a semi-circle configuration as shown in Fig. 3. They are directed away from the BS to suppress the line of sight (LOS) links and to ensure

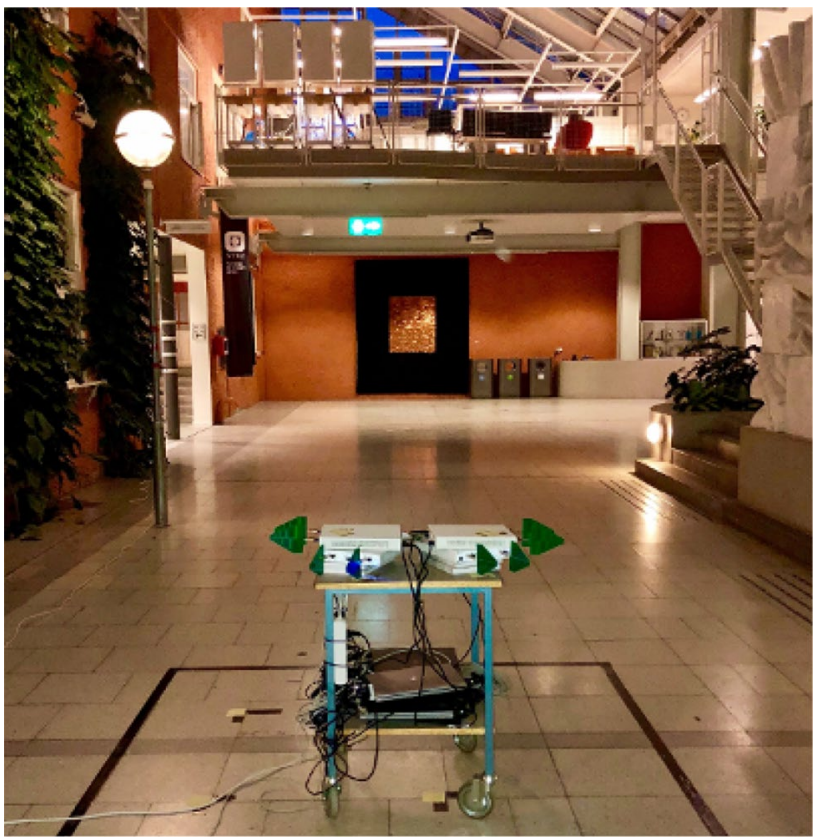

Fig. 3 Positioning of the base station on the balcony and the user equipment cart. The antennas are pointing away form the the balcony to illuminate non-line of sight links. Five physical locations in the black square were measured for each frequency band

reflected links are more accentuated. The approximate distance between the UE cart and the main reflectors at the end of the hall is around $30 \mathrm{~m}$. As shown in the campaign photos in Figs. 3 and 4, there exist many reflecting surfaces, such as concrete walls, window glasses, metal lamp posts and metal bars (at the end of the hallway) which form a rich scattering environment. For each frequency band, the UE cart was positioned along 5 pre-marked locations within $1 \mathrm{~m}$ diameter to obtain more sampling points of the environment.

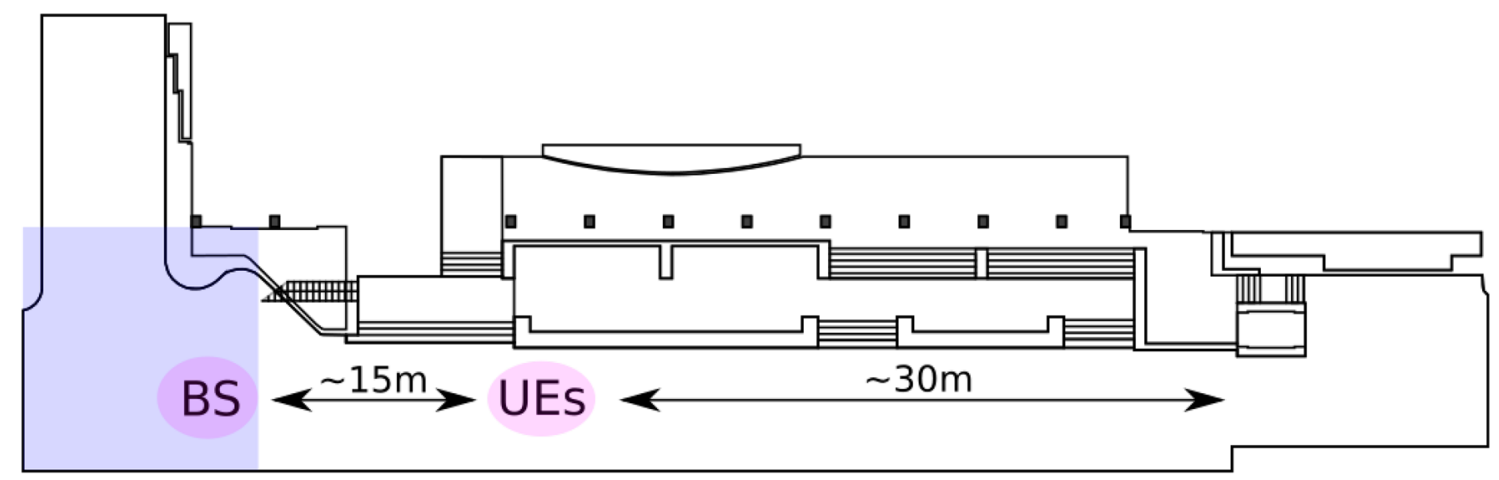

Fig. 2 Top down sketch of the scenario. The base station (BS) is placed on a balcony indicated with blue color. The user equipments (UEs) were placed at a distance of approximately $15 \mathrm{~m}$ with antenna orientation away from the BS subarrays 


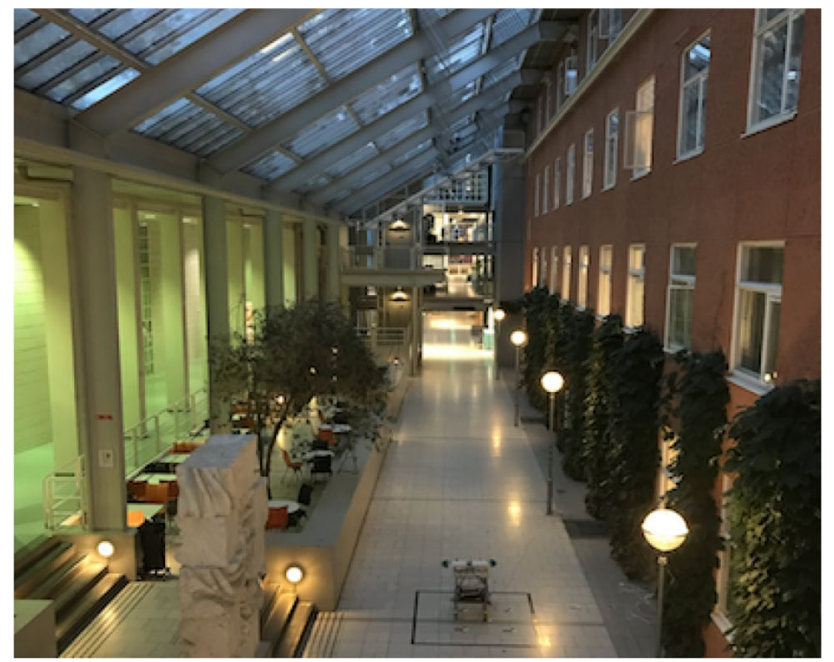

Fig. 4 Overview of the measurement scenario from the base station balcony as seen by the antenna array

\subsection{Channel Estimate Acquisition}

The system has $M$ antennas on the BS side, $K$ UEs and uses orthogonal frequency division multiplexing (OFDM) with 1200 usable subcarriers distributed over a $20 \mathrm{MHz}$ band. Each user $k$ transmits pilot symbols on a unique set of $F$ subcarriers (in total 100 subcarriers for each UE) during the channel estimate acquisition to ensure orthogonality between the pilot symbols. These symbols are used to estimate the channel coefficient $G_{m k}[f]$, with $m, k$ and $f$ as BS antenna index, user index and subcarrier index, respectively, by a least-squares method.

A received symbol $Y_{m f}^{\mathrm{BS}}$ in the UL can be written as

$Y_{m}^{\mathrm{BS}}[f]=\sum_{K} G_{m k}[f] X_{k}^{\mathrm{UE}}[f]+N_{m}[f]$

where $X_{k}^{\mathrm{UE}}[f]$ is the transmit symbol and $N_{m}[f]$ the noise at the receiver. Furthermore, the channel coefficient is divided into a large scale fading and shadowing factor $\left(\sqrt{\beta_{k}}\right)$ and a small scale fading factor $H_{m k}[f]$ :

$G_{m k}[f]=\sqrt{\beta_{k}} H_{m k}[f]$.

If $G_{m k}[f]$ is distributed according to a complex normal variable with zero mean and variance $\beta_{k}$, that is $\left.G_{m k}[f] \sim \mathbb{C N}\left(0, \beta_{k}\right)\right)$, then $H_{m k}[f] \sim \mathbb{C N}(0,1)$. The complex CTF coefficient $H_{m k}[f]$ is used in the rest of the manuscript to analyse properties of channel hardening. The delay domain representation is readily available via an inverse discrete Fourier transform (IDFT) along the frequency axis

$h_{m k}[n]=\underset{f}{\operatorname{IDFT}}\left\{H_{m k}[f]\right\}$

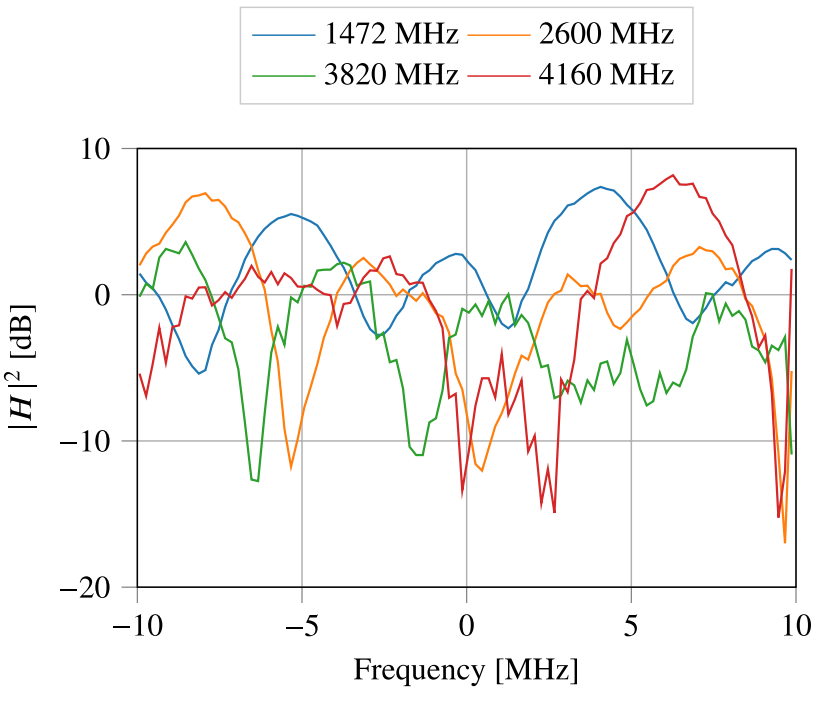

Fig. 5 Selected transfer functions of uplink channels between a single user and a single base station antenna are shown. Some subcarriers experience severe fading of more then $10 \mathrm{~dB}$

where $n$ denotes the delay bin. This representation corresponds directly to a tapped delay line.

\subsection{Measurement Results}

To represent the measured radio environment and to highlight the variations over the frequency range, we present one single CTF per frequency band in Fig. 5. Each CTF, normalized to its average power, demonstrates significant fading below $-10 \mathrm{~dB}$, as expected for a rich scattering environment.

Figure 6 represents normalized power delay profiles for all measured single input single output (SISO) channels by averaging over the realizations for all UEs and BS antennas. The channel confines most of the power in a delay window of $1 \mu \mathrm{s}$. Furthermore, 5-6 multipath components (MPCs) are clearly resolved for the observation bandwidth of the user. The response outside of $a \pm 1 \mu$ s window of the effective channel is considered to contain measurement noise and is therefore discarded. Additionally, artifacts of the IDFT at the delay border and noise contributions are hereby reduced.

\section{Effective Channel Concept}

In TDD massive MIMO systems, the UL channels between BS and UEs are estimated at the BS by using a set of orthogonal pilot symbols sent by each UE and received at each antenna element in the arrays, as depicted in Fig. 7a. Given reciprocity holds for the transmitter and receiver chains in the coherence bandwidth of the system, these channel estimates denoted by 


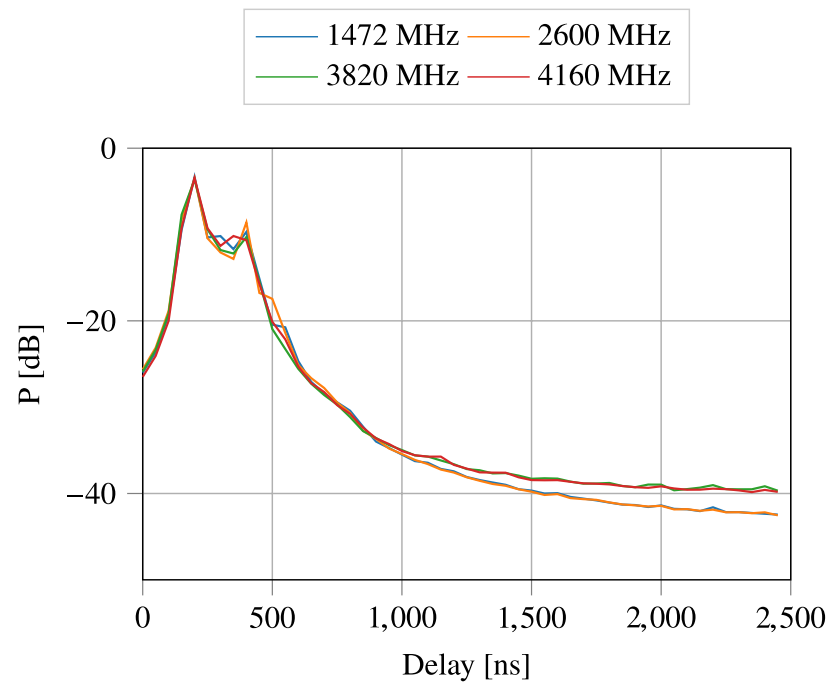

Fig. 6 Power delay profiles for the four frequency bands are shown. They were estimated by averaging over all base station antennas, users and measurement locations in a small area. Observed differences between the frequency bands are small. Hence, the small scale fading behaviour is not changing considerably between $1472 \mathrm{MHz}$ and $4160 \mathrm{MHz}$ in the reported scenario

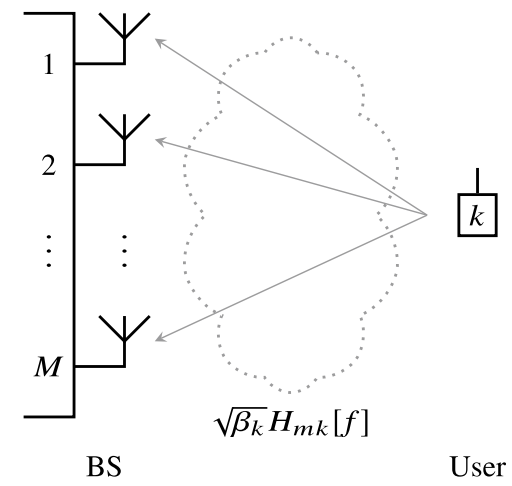

(a) Uplink Pilot Transmission

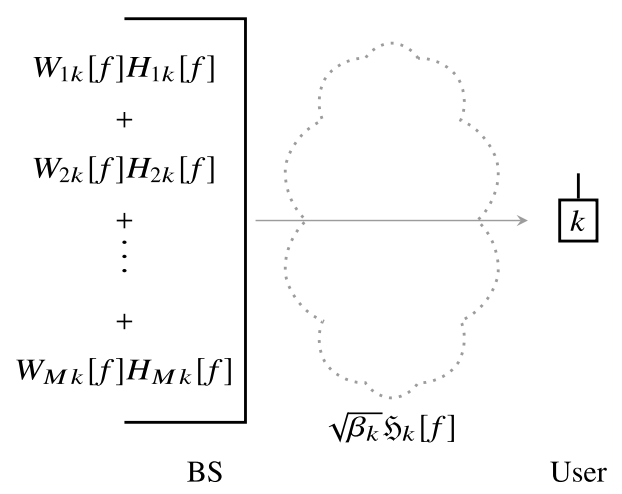

(b) Effective Downlink Channel

Fig. 7 Conceptual massive MIMO TDD operation. a Pilot signalling in the uplink to estimate $\mathfrak{S}_{m k}[f]$ at the base station. b Precoded downlink transmission with superposition at the user, who experiences the effective channel
$H_{m k}[f]$ are used to calculate the precoding weights at each antenna element. If no co-channel interference is assumed, the system can be considered as a multiple input single output (MISO) system and the effective channel perceived at the UE is formed by superposition of these individual channels. In other words, the UE, unaware of any beam forming or precoding, observes a DL SISO channel from the BS which is the effective channel.

As shown in Fig. 7b, from the UE's perspective, all the signals from the BS form an effective channel according to Eq. (4).

In our specific analysis, weighted sums of SISO CTF coefficients with freely chosen weights $W_{m k}[f]$ are used to form the effective channel CTF coefficients $\mathfrak{H}_{k}[f]$,

$\mathfrak{S}_{k}[f]=\sum_{M} W_{m k}[f] H_{m k}[f]$.

Therefore is the received signal at user $k$ in the DL

$$
\begin{aligned}
Y_{k}^{\mathrm{UE}}[f]= & \sqrt{\beta_{k}} \mathfrak{H}_{k}[f] X_{k}^{\mathrm{BS}}[f] \\
& +\sqrt{\beta_{k}} \sum_{M} H_{m k}[f] \sum_{l \neq k}^{K} W_{m l}[f] X_{l}^{\mathrm{BS}}[f] \\
& +N_{k}[f]
\end{aligned}
$$

where $Y_{k}^{\mathrm{UE}}[f]$ is the received DL signal at user $k$ and the DL symbol to user $k$ is $X_{l}^{\mathrm{BS}}[f]$. The second term constitutes the multiuser interference and $N_{k}[f]$ is the additive noise at user $k$. To illustrate the properties of the effective channel we choose the weights to be the complex conjugate of the CTF coefficients with a normalization of $\sqrt{M}$ to impose an average power constraint

$W_{m k}[f]=\frac{H_{m k}^{*}[f]}{\sqrt{M}}$.

This differs from maximum ratio transmission (MRT),

$W_{m k}^{M R T}[f]=\frac{H_{m k}^{*}[f]}{\sqrt{\sum_{M}\left|H_{m k}[f]\right|^{2}}}$

where the normalization is an instantaneous power constraint over the array [13]. For large $M$ the difference disappears as $\sum_{M}\left|H_{m k}[f]\right|^{2} \approx M E\left\{\left|H_{m k}[f]\right|^{2}\right\}=M$ due to the self averaging property of the large array. The chosen normalization for the matched filtering does maximize the average SNR in the DL and simplifies the time domain analysis in Sects. 4.1 and 4.2 as it directly transfers to time reversal precoding.

We take different subsets over the $M$ base station antennas, in order to form several effective channels and use them 
to determine how the different metrics behave for increasing number of antennas in the following section.

\section{Channel Hardening Metrics}

In the frequency domain, channel hardening is regarded as flat fading of the effective CTF over a large bandwidth. In the delay domain the channel hardening implies that the strong contributions of the effective channel impulse response (CIR) are confined to a single delay tap, with reliable tap power for most realizations. Since the rms delay spread of the effective CIR determines the necessity for an equalizer in the UE design, with sufficient channel hardening, the UE receiver could be simplified. The next three subsections describe the figures of merit for characterizing channel hardening in both delay and frequency domains.

\subsection{Power Variation of the Effective Channel}

Characterizing power variations between different subcarriers of the effective channel is a metric to assess the flatness of the CTF over the observed bandwidth. To allow for comparison between BS antenna subsets with different cardinality, the subcarrier power needs to be normalized by the expectation of its distribution, namely $\mathbb{E}\left\{\left|\mathfrak{S}_{k}[f]\right|^{2}\right\}=M+1$ The details of this derivation can be seen in "Appendix 1". The normalized power in the frequency domain is then

$\mathfrak{Q}_{k}[f]=\frac{\left|\mathfrak{S}_{k}[f]\right|^{2}}{M+1}$.

The distribution of $\mathfrak{Q}_{k}[f]$ allows to characterize the power level fluctuations in the DL a narrow band receiver (RX) will see over the frequency range. Hence, it allows to draw conclusions about the amount of fading that the link budget needs to take into account.

Figure 8 shows the empirical cumulative distribution functions (CDFs) of $\mathfrak{Q}_{k}[f]$. The combinations are formed from 1, 4, 16 and 64 antenna elements over all measured frequency bands, with channels drawn from similar subsets of consecutive close antenna elements in the subarrays. The observed statistics of the channel is practically the same in the range $1.5-4.2 \mathrm{GHz}$. Furthermore, the variation of $\mathfrak{Q}_{k}[f]$ reduces with increasing $M$. Considering the link budget, channel hardening would reduce the fading to $2 \mathrm{~dB}$ for $90 \%$ of the observed effective channels with 64 antennas at the BS.

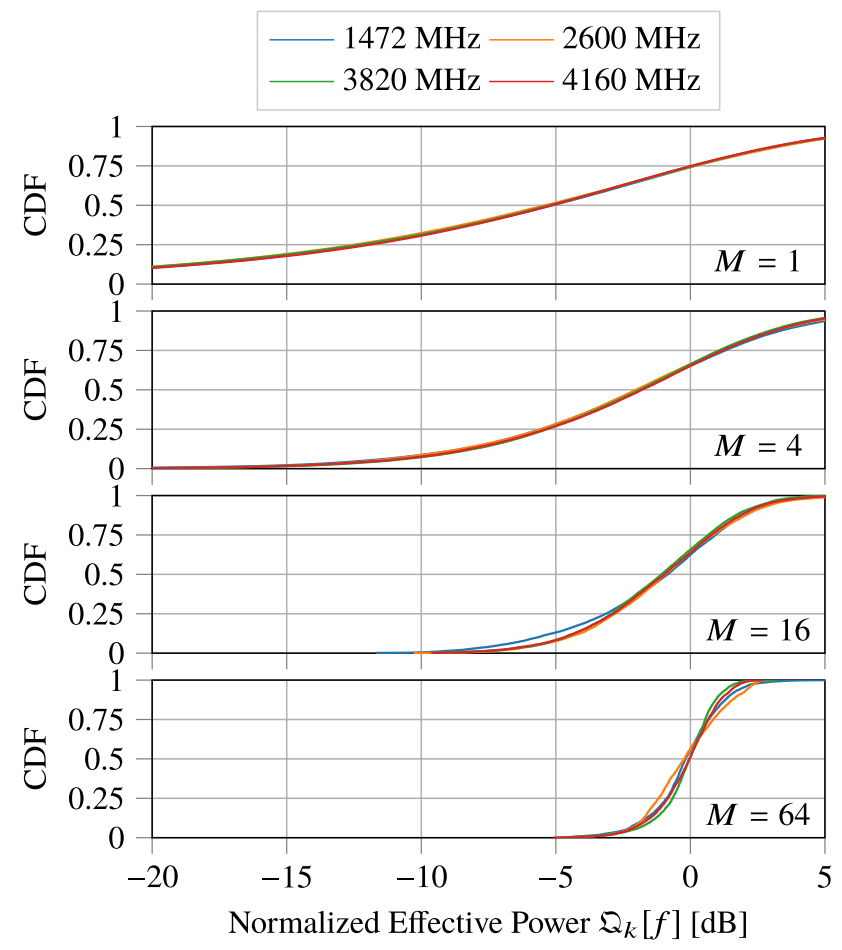

Fig. 8 Empirical cumulative distribution functions for the normalized effective subcarrier power are shown. Fading has a lesser impact the higher the number of used BS antennas in the DL. Combination of 64 transmit signals at the receiver reduce the fading to less then $2 \mathrm{~dB}$ in $90 \%$ of the realisations for all four frequency bands

\subsection{Effective PDP and Coherence Bandwidth}

In this section, first we derive an analytical form for the effective power delay profile (PDP) as a function of the PDP of the UL channels. Weights given by Eq. (6) are used to implement time reversal precoding in the DL. The frequency correlation is calculated as the Fourier transform of the effective PDP, then coherence bandwidth can be obtained as a metric for evaluation of the effective channel.

Under the assumption of independent and equally distributed channels for user $k$ at all antenna elements, the channel can be described as complex normal distributed $h_{m k}[n] \sim \mathbb{C N}\left(0, p_{k}[n]\right)$ where $p_{k}[n]=\mathbb{E}\left\{\left|h_{m k}[n]\right|^{2}\right\}$ is the PDP for all the channels from user $k$ to the array. With the normalization of path loss and large scale fading the average channel gain is $\sum p_{k}[n]=1$. The use of the weights from Eq. (6) in Eq. (4) corresponds to applying the matched filter in the time domain,

$w_{m k}[n]=h_{m}^{*}[-n] / \sqrt{M}$, 


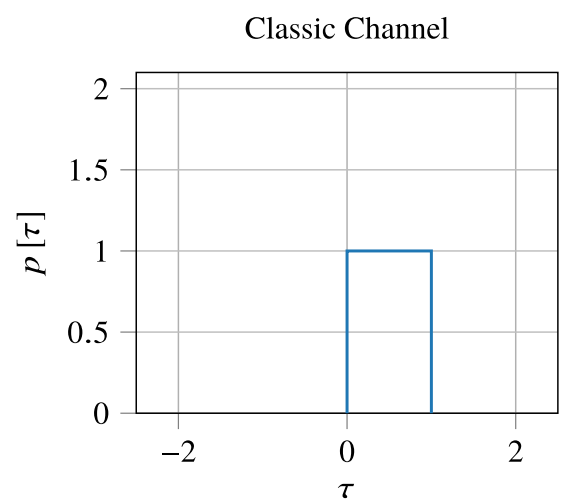

(a)

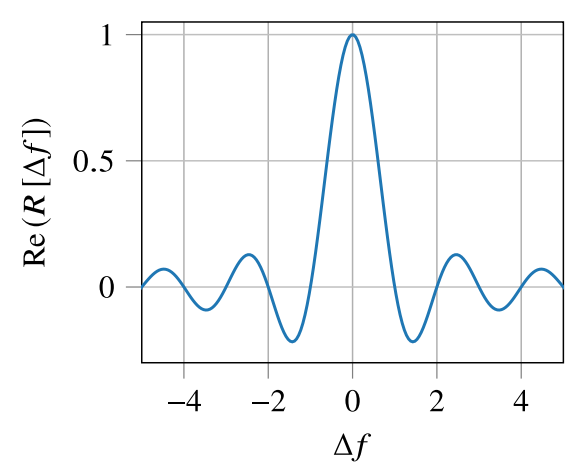

(d)

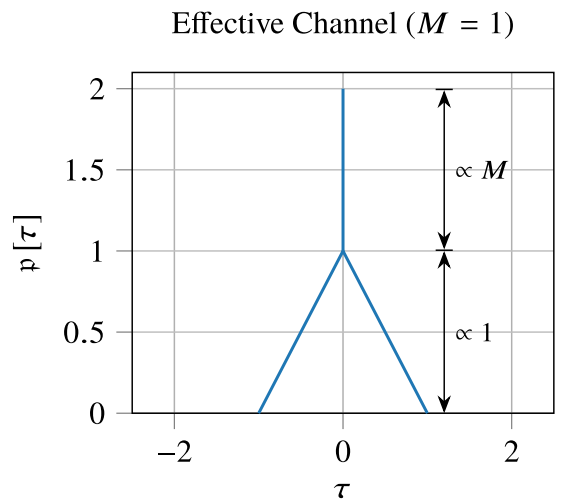

(b)

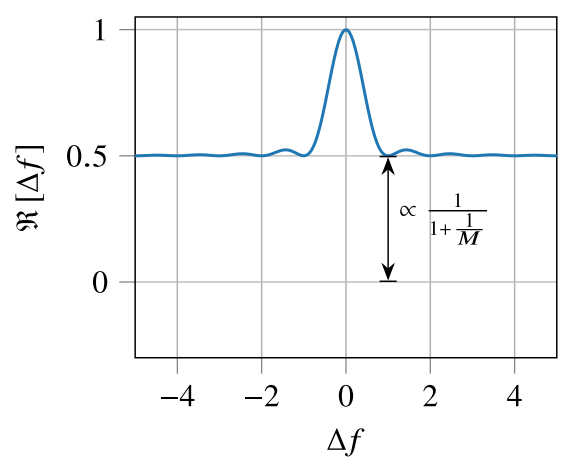

(e)
Effective Channel $(M=4)$

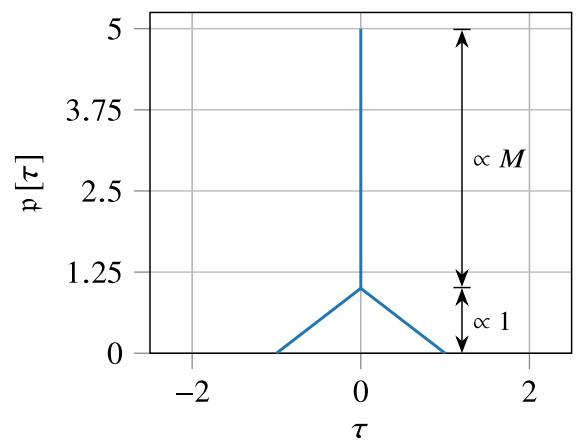

(c)

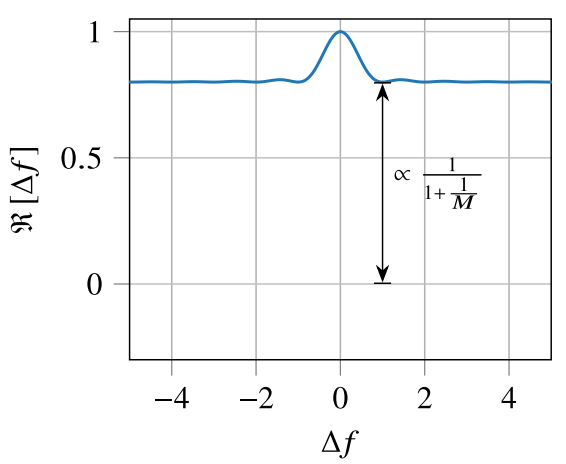

(f)
Fig. 9 The effective PDPs of an original square PDPs and the Fourier related frequency correlation functions are shown in $(\mathbf{a}-\mathbf{c})$ and (d-f), respectively. The effective channels has a scaling behaviour of

which is time-reversal precoding [3]. The normalization keeps the average output power from the array independent of $M$ when these weights are used in a precoding filter. An instantaneous power constraint would give the normalization with $\sqrt{\sum_{M} \sum_{n}\left|h_{m k}[n]\right|^{2}}$, which for large $M$ approximately is $\sqrt{M}$ as

$$
\sum_{M} \sum_{n}\left|h_{m k}[n]\right|^{2} \approx M \mathbb{E}\left\{\left|h_{m k}[n]\right|^{2}\right\}=M
$$

The effective channel in the delay domain becomes

$\mathfrak{h}_{k}[n]=\sum_{M} w_{m k}[n] * h_{m k}[n]$.

The corresponding effective PDP is derived in "Appendix 2" and is given as

$\mathfrak{p}_{k}[n]=p_{k}[-n] * p_{k}[n]+\delta_{n} M$.

The temporal focusing is visualized in Fig. 9a-c. It can be seen that the ratio of the amount of energy at the zero delay tap to the amount of energy at non-zero taps increases the PDP for different numbers of antennas. The increasing peak in the PDP for larger $M$ corresponds to an increasing lower bound on the frequency correlation, which approaches flat fading for large $M$

as the number of combined elements grows. The frequency correlation function for all the channels for user $k$ is denoted $R_{k}[\Delta f]$ and is the Fourier transform of the PDP $p_{k}[n][5]$. The correlation function $\mathfrak{R}_{k}[\Delta f]$ for the effective channel $\mathfrak{h}_{k}[n]$ is hence the Fourier transform of $\mathfrak{p}_{k}[n]$. The PDP $\mathfrak{p}_{k}[n]$ consist of two terms. The convolution $p_{k}[-n] * p_{k}[n]$ corresponds in the frequency domain to the absolute square of the frequency correlation, that is $\left|R_{k}[\Delta f]\right|^{2}$. The peak $\delta_{n} M$ in Eq. (12) at zero lag corresponds to an offset for all frequencies. Hence, with a normalization so that $\mathfrak{R}_{k}[0]=1$ we obtain

$\mathfrak{R}_{k}[\Delta f]=\frac{\left|R_{k}[\Delta f]\right|^{2}+M}{1+M}$.

The link between the PDP and the frequency correlation is illustrated in Fig. 9. Because the correlation function $R_{k}[\Delta f]$ is bounded as $0 \leq\left|R_{k}[\Delta f]\right|^{2} \leq 1$, the effective frequency correlation becomes bounded as

$\frac{1}{1+1 / M} \leq \Re_{k}[\Delta f] \leq 1$ 
The large offset caused by the matched filtering limits the variability of the frequency correlation function. Even with just one antenna, matched filtering reduces the maximum range of $\Re_{k}[\Delta f]$ to $3 \mathrm{~dB}$ as illustrated in Fig. 9e. For four antennas it is $1 \mathrm{~dB}$ and for 8 antennas it is $0.5 \mathrm{~dB}$. Hence, the common $3 \mathrm{~dB}$ coherence bandwidth measure can not be applied for the effective channel using matched filtering, as the coherence bandwidth will become the whole bandwidth when using two or more antennas. The channel hardening is here seen as the reduction in frequency selectivity, which results in a coherence bandwidth equal to almost the full bandwidth. Using an instantaneous power constraint would even further reduce the variability.

As the variability of the frequency response $\mathfrak{S}_{k}[f]$ reduces with $M$ the coherence bandwidth will increase simply because all the subcarriers will have just minor differences in amplitude around a dominating average.

\subsection{The Effective Delay Spread}

The effective PDP $\mathfrak{p}[n]$ will have an effective delay spread given by

$\hat{\tau}_{r m s}=\Delta \tau \sqrt{\frac{\sum_{n=-\infty}^{\infty} n^{2} \mathfrak{p}[n]}{\sum_{n=-\infty}^{\infty} \mathfrak{p}[n]}}$,

where $\Delta \tau$ is the duration between samples. To be able to study the variability of temporal focusing of the matched filtering for the realizations we use the instantaneous delay spread, adapted from [4],

$\hat{\tau}_{r m s_{k}}^{i}=\Delta \tau \sqrt{\frac{\sum_{n=-\infty}^{\infty} n^{2}\left|\mathfrak{h}_{k}[n]\right|^{2}}{\sum_{n=-\infty}^{\infty}\left|\mathfrak{h}_{k}[n]\right|^{2}}}$,

where the expectations in Eq. (15) are exchanged with realizations. Channel hardening manifests as a reduction in variability whereas temporal focusing results in an over all reduction of this measure. Figure 10 shows the empirical $\mathrm{CDF}$ of the instantaneous rms delay spread for all channel realizations. Both the value and the variability of $\hat{\tau}_{r m s_{k}}^{i}$ reduce with $M$. For large $M$ this will make it possible to have low complexity receivers in the DL as the channel effectively becomes a single tap channel.

The ratio between the effective delay spread $\hat{\tau}_{r m s}$ and the delay spread before combining $\tau_{r m s}$, derived in "Appendix 3", can be expressed as a function of the number of antennas $M$ as

$\frac{\hat{\tau}_{r m s}}{\tau_{r m s}}=\sqrt{\frac{2}{1+M}}$.

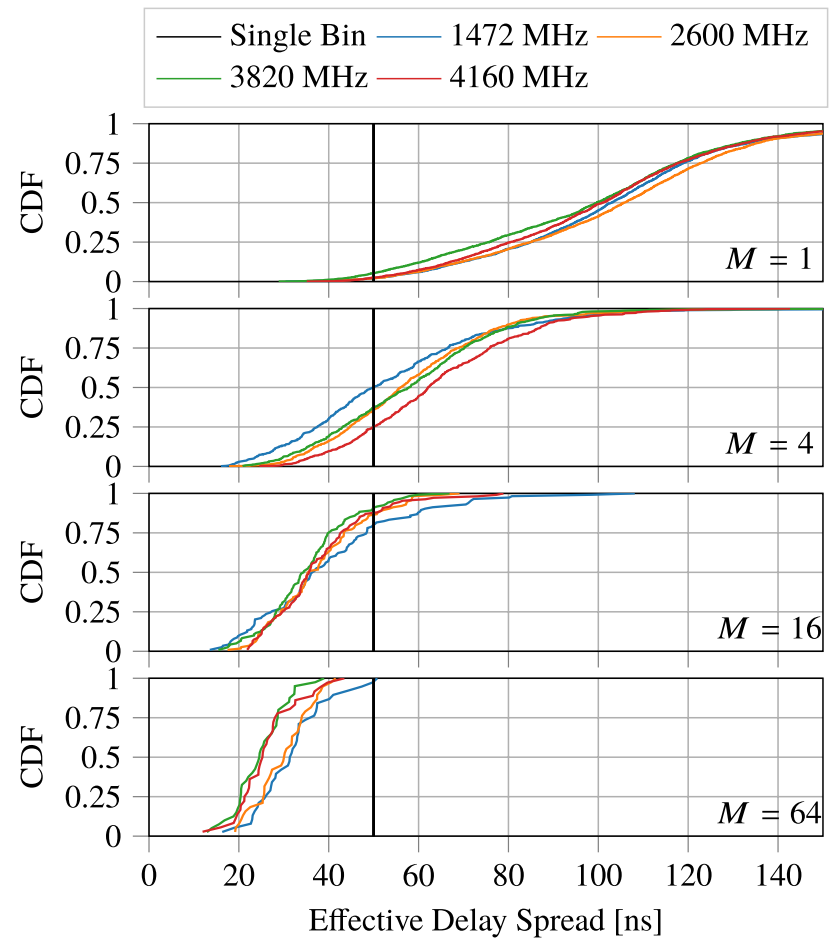

Fig. 10 Empirical CDFs for the effective delay spread. Both the mean as well as the variance are decreasing for an increasing number of antennas, demonstrating temporal focusing and channel hardening. A base station with 64 antennas could shrink the delay spread to fit into a single delay bin allowing for a simplified receiver at the user side

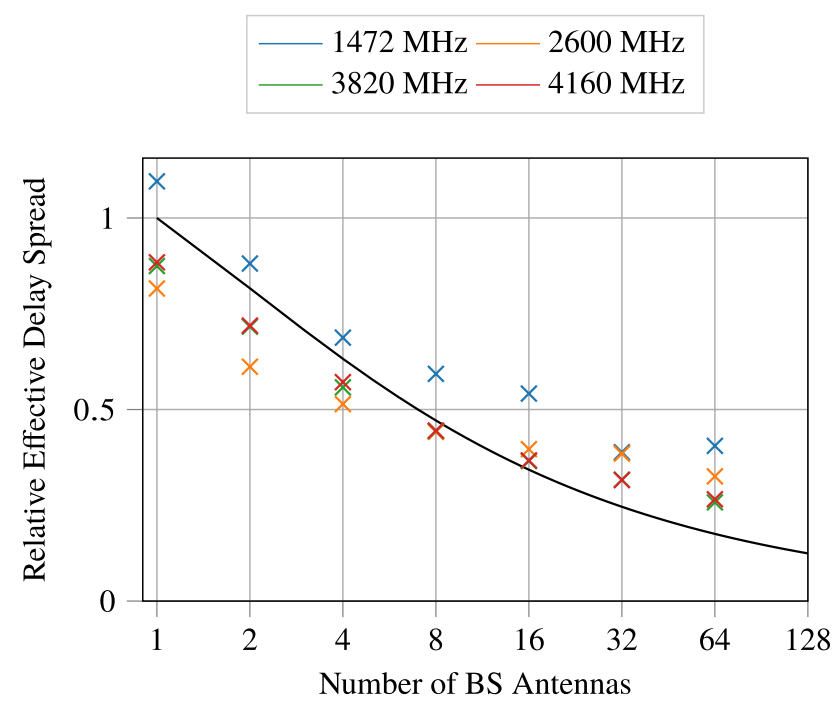

Fig. 11 The ratio of effective delay spread and the delay spread of the channel as a function of antennas used for combining is shown. The theoretical scaling is drawn with a black line (Eq. (17)). A system with 8 antennas at the base station could reduce the delay spread by a factor of two and the effect has diminishing returns for larger systems 
For a single antenna the matched filtering does not change the delay spread. Figure 11 shows the ratio $\hat{\tau}_{r m s} / \tau_{r m s}$ for the empirical delay spread from the measurements together with the ratio given by Eq. (17). The delay spread converges slower to a single tap than the coherence bandwidth approaches the full bandwidth when increasing the number of combined antennas. Using 7 antennas will halve the delay spread whereas 16 antennas reduce it by approximately a third and 32 by a fourth.

The observed channel hardening is the focusing of the signal in the delay domain resulting in a smaller delay spread. In addition, the variability of the delay spread in the realizations reduce with increasing numbers of combined antennas as seen in Fig. 10.

\section{Conclusion}

Measured data from a quasi-static indoor radio environment over four frequency bands in the range from 1.4 to $4.2 \mathrm{GHz}$ are used to study channel hardening properties in massive MIMO when using time-reversal precoding. The measurements contain 40 single user realizations against a 64 element antenna array BS to elucidate channel hardening in both the delay and the frequency domain. The observed statistical properties for the channels are practically the same over the studied frequency bands. The coherence bandwidth is demonstrated to have limited merit as a measure for the effective DL channel. The rms delay spread and the normalized subcarrier power of the effective radio channel are described as physically motivated figures of merit for low complexity single tap receivers

Acknowledgements ReRaNP is supported by the Norwegian Research Council within the National Financing Initiative for Research Infrastructure. Further support was provided by Telenor ASA within the project Testing RAN Options for 5G.

Funding Funding was provided by Norges Forskningsråd (Grant No. 245699).

Open Access This article is distributed under the terms of the Creative Commons Attribution 4.0 International License (http://creativeco mmons.org/licenses/by/4.0/), which permits unrestricted use, distribution, and reproduction in any medium, provided you give appropriate credit to the original author(s) and the source, provide a link to the Creative Commons license, and indicate if changes were made.

\section{Expectation of the Effective Channel Power in the Frequency Domain}

Taking the small scale coefficients $H_{m}[f] \sim \mathbb{C} \mathcal{N}(0,1)$ from a user to antenna $m$ on subcarrier $f$ and normalizing the matched filter weights $W_{m}[f]$ with $\sqrt{M}$ allows us to write the effective channel with dropped user dependency in Eq. (4) as follows:

$$
\begin{aligned}
\mathfrak{H}[f] & =\sum_{M} W_{m}[f] H_{m}[f]=\frac{1}{\sqrt{M}} \sum_{M} H_{m}^{*}[f] H_{m}[f] \\
& =\frac{1}{\sqrt{M}} \sum_{M}\left|H_{m}[f]\right|^{2} .
\end{aligned}
$$

As $\mathbb{E}\left\{\left|H_{m}[f]^{2}\right|\right\}=1$ and $\mathbb{E}\left\{\left|H_{m}[f]\right|^{4}\right\}=2$ for a complex circular Gaussian variable with unit variance [17], using the Kronecker delta function $\delta_{i j}$, the effective channel power is obtained as

$$
\begin{aligned}
\mathbb{E} & \left\{|\mathfrak{H}[f]|^{2}\right\} \\
& =\frac{1}{M} \sum_{m=1}^{M} \sum_{l=1}^{M} \mathbb{E}\left\{\left|H_{m}[f]\right|^{2}\left|H_{l}[f]\right|^{2}\right\} \\
& =\frac{1}{M} \sum_{m=1}^{M} \sum_{l=1}^{M}\left[\begin{array}{c}
\left(1-\delta_{m l}\right) \mathbb{E}\left\{\left|H_{m}[f]\right|^{2}\right\} \mathbb{E}\left\{\left|H_{l}[f]\right|^{2}\right\} \\
+\delta_{m l} \mathbb{E}\left\{\left|H_{m}[f]\right|^{4}\right\}
\end{array}\right] \\
& =\frac{1}{M} \sum_{m=1}^{M} \sum_{l=1}^{M}\left(1-\delta_{m l}\right)+2 \delta_{m l} \\
& =\frac{1}{M} \sum_{m=1}^{M} \sum_{l=1}^{M}\left(1+\delta_{m l}\right)=\frac{1}{M}\left(M^{2}+M\right)=M+1 .
\end{aligned}
$$

Hence, the expectation of the effective channel power and the SNR in the DL scales with $M+1$, if the weights are normalized with $\sqrt{M}$.

\section{Power Delay Profile of the Effective Channel}

Examining the single user case the user index is neglected. The channel from UE to antenna $m$ is given as $h_{m} \sim \mathbb{C N}(0, p[n])$, where $p[n]$ is the power delay profile assumed the same over the whole array. The average channel gain is one, that is $\sum_{n=0}^{\infty} p[n]=1$. With the matched filtering $w_{m}[n]=h_{m}^{*}[-n] / \sqrt{M}$ the PDP for an effective channel is the expectation of the absolute square of the effective channel impulse response,

$\mathfrak{p}[n]=\mathbb{E}\left\{|\mathfrak{h}[n]|^{2}\right\}=\mathbb{E}\left\{\frac{1}{M}\left|\sum_{m=1}^{M} h_{m}^{*}[-n] * h_{m}[n]\right|^{2}\right\}$

As the channels $h_{m}[n]$ are causal, the matched filtering of channel $m$, that is the convolution, can be expressed as 


$$
\begin{aligned}
\mathfrak{h}_{m}[n] & =w_{m}[n] * h_{m}[n]=\frac{h_{m}^{*}[-n]}{\sqrt{M}} * h_{m}[n] \\
& =\frac{1}{\sqrt{M}} \sum_{v=-\infty}^{\infty} h_{m}^{*}[-v] h_{m}[n-v] \\
& =\frac{1}{\sqrt{M}} \sum_{\nu=-\infty}^{\infty} h_{m}^{*}[-v] h_{m}[n-v] \\
& =\frac{1}{\sqrt{M}} \sum_{v=0}^{\infty} h_{m}^{*}[v] h_{m}[n+v] .
\end{aligned}
$$

Here $\mathfrak{h}_{m}[n]$ is the effective channel for a single antenna $m$ after matched filtering. The expectation is

$\mathbb{E}\left\{\mathfrak{h}_{m}[n]\right\}=\frac{1}{\sqrt{M}} \sum_{\nu=0}^{\infty} \underbrace{\mathbb{E}\left\{h_{m}^{*}[\nu] h_{m}[n+\nu]\right\}}_{\delta_{n} p[\nu]}=\frac{\delta_{n}}{\sqrt{M}}$

where we use that the sum power in the PDP is one and the corresponding effective PDP for antenna $m$ is

$$
\begin{aligned}
\mathfrak{p}_{m}[n] & =\mathbb{E}\left\{\left|\mathfrak{h}_{m}[n]\right|^{2}\right\} \\
& =\mathbb{E}\left\{\frac{1}{M}\left|\sum_{\nu=0}^{\infty} h_{m}^{*}[\nu] * h_{m}[n+v]\right|^{2}\right\} \\
& =\frac{1}{M} \sum_{\nu=0}^{\infty} \sum_{\mu=0}^{\infty} \mathbb{E}\left\{h_{m}^{*}[\nu] h_{m}[n+v] h_{m}[\mu] h_{m}^{*}[n+\mu]\right\} .
\end{aligned}
$$

The expectation in the sum can be broken down in four terms, each a combination of $n=0, n \neq 0$ and $\nu=\mu, \nu \neq \mu$. Using $\delta_{i j}$ and the unit impulse $\delta_{i}$ to separate the terms we obtain

$$
\begin{aligned}
& \mathbb{E}\left\{h_{m}^{*}[\nu] h_{m}[n+\nu] h_{m}[\mu] h_{m}^{*}[n+\mu]\right\} \\
&=\left(1-\delta_{n}\right)\left(1-\delta_{v \mu}\right) \cdot 0 \\
&+\delta_{n}\left(1-\delta_{\nu \mu}\right) \underbrace{\mathbb{E}\left\{\left|h_{m}[v]\right|^{2}\right\}}_{p[v]} \underbrace{\mathbb{E}\left\{\left|h_{m}[\mu]\right|^{2}\right\}}_{p[\mu]} \\
&+\left(1-\delta_{n}\right) \delta_{v \mu} \underbrace{\mathbb{E}\left\{\left|h_{m}[v]\right|^{2}\right\}}_{p[v]} \underbrace{\mathbb{E}\left\{\left|h_{m}[n+\nu]\right|^{2}\right\}}_{p[n+\nu]} \\
&+\delta_{n} \delta_{\nu \mu} \underbrace{\mathbb{E}\left\{\left|h_{m}[v]\right|^{4}\right\}}_{2 p^{2}[v]}
\end{aligned}
$$

Using the WSSUS model, taps corresponding to different delays are independent and hence the first term is zero.
Gathering the terms containing either $\delta_{v \mu}$ or $\delta_{n}$ in Eq. (24) the expectation becomes

$$
\begin{array}{r}
\mathbb{E}\left\{h_{m}^{*}[v] h_{m}[n+v] h_{m}[\mu] h_{m}^{*}[n+\mu]\right\} \\
\quad=\delta_{v \mu} p[v] p[n+v]+\delta_{n} p[v] p[\mu]
\end{array}
$$

Using this in Eq. (23) the effective PDP for channel $m$ is obtained as

$$
\begin{aligned}
\mathfrak{p}_{m}[n] & =\frac{1}{M} \sum_{\nu=0}^{\infty} \sum_{\mu=0}^{\infty}\left(\delta_{\nu \mu} p[v] p[n+\nu]+\delta_{n} p[v] p[\mu]\right) \\
& =\frac{1}{M} \sum_{\nu=0}^{\infty} p[v] p[n+\nu]+\frac{\delta_{n}}{M} \underbrace{\sum_{v=0}^{\infty} p[v]}_{1} \underbrace{\sum_{\mu=0}^{\infty} p[\mu]}_{1} \\
& =\frac{1}{M}\left(p[-n] * p[n]+\delta_{n}\right)
\end{aligned}
$$

Using Eq. (22) the expectation of the effective channel is

$\mathbb{E}\{\mathfrak{h}[n]\}=\mathbb{E}\left\{\sum_{m=1}^{M} \mathfrak{h}_{m}[n]\right\}=\sqrt{M} \delta_{n}$

The effective PDP after combining is

$\mathfrak{p}[n]=\mathbb{E}\left\{\left|\sum_{m=1}^{M} \mathfrak{h}_{m}[n]\right|^{2}\right\}=\sum_{m=1}^{M} \sum_{l=1}^{M} \mathbb{E}\left\{\mathfrak{h}_{m}[n] \mathfrak{h}_{l}^{*}[n]\right\}$

Approaching the expectation in the same manner as in Eq. (24) we obtain

$$
\begin{aligned}
\mathbb{E}\left\{\mathfrak{h}_{m}[n] \mathfrak{h}_{l}^{*}[n]\right\}= & \left(1-\delta_{n}\right)\left(1-\delta_{m l}\right) \cdot 0 \\
& +\delta_{n}\left(1-\delta_{m l}\right) \underbrace{\mathbb{E}\left\{\mathfrak{h}_{m}[0]\right\}}_{1 / \sqrt{M}} \underbrace{\mathbb{E}\left\{\mathfrak{h}_{l}[0]\right\}}_{1 / \sqrt{M}} \\
& +\delta_{m l} \underbrace{\mathbb{E}\left\{\left|\mathfrak{h}_{m}[n]\right|^{2}\right\}}_{\mathfrak{p}_{m}[n]}
\end{aligned}
$$

which when introduced in Eq. (28) together with Eq. (26) result in

$$
\begin{aligned}
\mathfrak{p}[n] & =\sum_{m=1}^{M} \sum_{l=1}^{M}\left(\delta_{m l} \mathfrak{p}_{m}[n]+\frac{\delta_{n}\left(1-\delta_{m l}\right)}{M}\right) \\
& =\sum_{m=1}^{M} \mathfrak{p}_{m}[n]+(M-1) \delta_{n} \\
& =p[-n] * p[n]+M \delta_{n} .
\end{aligned}
$$


The effective PDP has the shape of the convolution of the underlying PDP with it's reverse with an additional $M \delta_{n}$ peak in the middle, formed by the coherent summing. The gain of the effective PDP is

$$
\begin{aligned}
\sum_{n=-\infty}^{\infty} \mathfrak{p}[n] & =\sum_{n=-\infty}^{\infty} \sum_{v=0}^{\infty} p[v] p[n+v]+M \sum_{n=-\infty}^{\infty} \delta_{n} \\
& =\underbrace{\sum_{v=0}^{\infty} p[v]}_{=1} \underbrace{\sum_{n=-\infty}^{\infty} p[n+v]}_{=1}+M=1+M
\end{aligned}
$$

Of special interest is the center tap $\mathfrak{p}[0]$ which is obtained from Eq. (29) and that can be bounded as

$M<\mathfrak{p}[0]=\sum_{v=0}^{\infty} p^{2}[v]+M \leq 1+M$.

where we use that all the terms in the sum are either zero or positive and that $p^{2}[n] \leq p[n] \leq 1$.

\section{Delay Spread of the Effective Channel}

As the effective PDP is symmetric around zero the average delay is zero. The squared delay spread for the effective channel is therefore given by

$$
\begin{aligned}
\hat{\tau}_{r m s}^{2} & =\Delta \tau^{2} \frac{\sum_{n=-\infty}^{\infty} n^{2} \mathfrak{p}[n]}{\sum_{n=-\infty}^{\infty} \mathfrak{p}[n]} \\
& =\frac{\Delta \tau^{2}}{M+1} \sum_{n=-\infty}^{\infty} n^{2} p[-n] * p[n]
\end{aligned}
$$

where we insert the result from Eq. (29) and use Eq. (30). The delay spread of the channels is given by

$$
\begin{aligned}
& \bar{\tau}=\Delta \tau \frac{\sum_{n=0}^{\infty} n p[n]}{\sum_{n=0}^{\infty} p[n]}=\Delta \tau \sum_{n=0}^{\infty} n p[n] \\
& \tau_{r m s}^{2}=\Delta \tau^{2} \frac{\sum_{n=0}^{\infty} n^{2} p[n]}{\sum_{n=-\infty}^{\infty} p[n]}-\bar{\tau}^{2}=\Delta \tau^{2} \sum_{n=0}^{\infty} n^{2} p[n]-\bar{\tau}^{2}
\end{aligned}
$$

For notational convenience we set $\Delta \tau=1$ and henceforth express the average delay and delay spread in samples. The effective delay spread can then be expressed as

$$
\begin{aligned}
\hat{\tau}_{r m s}^{2} & =\frac{1}{M+1} \sum_{n=-\infty}^{\infty} n^{2} \sum_{\nu=0}^{\infty} p[\nu] p[n+v] \\
& =\frac{1}{\mu=n+v} \sum_{\nu=0}^{\infty} p[\nu] \sum_{\mu=-\infty}^{\infty}(\mu-v)^{2} p[\mu],
\end{aligned}
$$

where a variable substitution in the second equality make it possible to separate the sums. The second sum can be evaluated as

$$
\begin{aligned}
\sum_{\mu=0}^{\infty}(\mu-v)^{2} p[\mu]= & \underbrace{\sum_{\mu=-\infty}^{\infty} \mu^{2} p[\mu]}_{\tau_{r m s}^{2}-\bar{\tau}^{2}}-2 \nu \underbrace{\sum_{\mu=-\infty}^{\infty} \mu p[\mu]}_{\bar{\tau}} \\
& +v^{2} \underbrace{\sum_{\mu=-\infty}^{\infty} p[\mu]}_{=1}
\end{aligned}
$$

which introduced back in Eq. (36) and performing the same type of evaluation of the sum as above result in

$$
\begin{aligned}
\hat{\tau}_{r m s}^{2} & =\frac{1}{M+1} \sum_{\nu=0}^{\infty} p[v]\left(\tau_{r m s}^{2}-\bar{\tau}^{2}-2 \nu \bar{\tau}+v^{2}\right) \\
& =\frac{2 \tau_{r m s}^{2}}{M+1}
\end{aligned}
$$

The ratio of the delay spread between the original channels and the effective channel after MRC or MRT is hence

$\frac{\hat{\tau}_{r m s}}{\tau_{r m s}}=\sqrt{\frac{2}{1+M}}$.

With a single antenna and matched filtering the delay spread is unaffected.

\section{References}

1. (2017) Introduction to the NI MIMO Prototyping System Hardware. http://www.ni.com/white-paper/53197/en/

2. E. Björnson, E. G. Larsson and T. L. Marzetta, Massive MIMO: ten myths and one critical question, Vol. 54, No. 2, pp. 114-123, 2016. https://doi.org/10.1109/MCOM.2016.7402270.

3. H. El-Sallabi, P. Kyritsi, A. Paulraj and G. Papanicolaou, Experimental investigation on time reversal precoding for space-time focusing in wireless communications, IEEE Transactions on Instrumentation and Measurement, Vol. 59, No. 6, pp. 15371543, 2010. https://doi.org/10.1109/TIM.2009.2024339.

4. M. P. Fitton, A. R. Nix, M. A. Beach. Evaluation of metrics for characterising the dispersion of the mobile channel. In Vehicular Technology Conference-VTC, IEEE, pages 1418-1422, 1996.

5. B. Fleury, An uncertainty relation for WSS processes and its application to WSSUS systems, IEEE Transactions on Communications, Vol. 44, No. 12, pp. 1632-1634, 1996. https://doi. org/10.1109/26.545890.

6. G. Ghiaasi, J. Abraham, E. Eide, T. Ekman. Measured channel hardening in an indoor multiband scenario. In 2018 IEEE 29th Annual International Symposium on Personal, Indoor, 
and Mobile Radio Communications (PIMRC), Bologna, Italy, pages 1-6, 2018. https://doi.org/10.1109/PIMRC.2018.85810 26, arXiv: 1812.05463.

7. J. C. Guey, L. Larsson. Modeling and evaluation of MIMO systems exploiting channel reciprocity in TDD mode. In IEEE 60th Vehicular Technology Conference (VTC2004-Fall), IEEE, pages 4265-4269, 2004. https://doi.org/10.1109/VETECF.2004.14048 83.

8. S. Gunnarsson, J. Flordelis, L. V. D. Perre, F. Tufvesson. Channel hardening in massive MIMO-a measurement based analysis. In 2018 IEEE 19th International Workshop on Signal Processing Advances in Wireless Communications (SPAWC), pages 1-5, 2018. https://doi.org/10.1109/SPAWC.2018.84459 25, arXiv: 1804.01690 .

9. P. Harris, S. Zhang, M. Beach, E. Mellios, A. Nix, S. Armour, A. Doufexi, K. Nieman, N. Kundargi. LOS throughput measurements in real-time with a 128-antenna massive MIMO testbed. In 2016 IEEE Global Communications Conference (GLOBECOM), IEEE, pages 1-7, 2016. https://doi.org/10.1109/GLOCO M.2016.7841965.

10. P. Harris, S. Malkowsky, J. Vieira, E. Bengtsson, F. Tufvesson, W. B. Hasan, L. Liu, M. Beach, S. Armour and O. Edfors, Performance characterization of a real-time massive MIMO system with LOS mobile channels, IEEE Journal on Selected Areas in Communications, Vol. 35, No. 6, pp. 1244-1253, 2017. https:// doi.org/10.1109/JSAC.2017.2686678.

11. B. M. Hochwald, T. L. Marzetta and V. Tarokh, Multipleantenna channel hardening and its implications for rate feedback and scheduling, IEEE Transactions on Information Theory, Vol. 50, No. 9, pp. 1893-1909, 2004. https://doi.org/10.1109/ TIT.2004.833345

12. E. Larsson, O. Edfors, F. Tufvesson and T. Marzetta, Massive MIMO for next generation wireless systems, Vol. 52, No. 2, pp. 186-195, 2014. https://doi.org/10.1109/MCOM.2014.6736761.

13. T. K. Y. Lo. Maximum ratio transmission. In 1999 IEEE International Conference on Communications, vol. 2, pages 13101314, 1999. https://doi.org/10.1109/ICC.1999.765552.

14. S. Malkowsky, J. Vieira, L. Liu, P. Harris, K. Nieman, N. Kundargi, I. C. Wong, F. Tufvesson, V. Owall and O. Edfors, The world's first real-time testbed for massive MIMO: design, implementation, and validation, IEEE Access, Vol. 5, pp. 90739088, 2017. https://doi.org/10.1109/ACCESS.2017.2705561.

15. A. O. Martinez, E. De Carvalho, J. O. Nielsen. Massive MIMO properties based on measured channels: channel hardening, user decorrelation and channel sparsity. In 2016 50th Asilomar Conference on Signals, Systems and Computers, IEEE, pages 18041808, 2016. https://doi.org/10.1109/ACSSC.2016.7869694.

16. S. Payami, F. Tufvesson. Delay spread properties in a measured massive MIMO system at 2.6 GHz. In 2013 IEEE 24th Annual International Symposium on Personal, Indoor, and Mobile Radio Communications (PIMRC), IEEE, pages 53-57, 2013. https://doi. org/10.1109/PIMRC.2013.6666103.

17. I. Reed, On a moment theorem for complex Gaussian processes, IRE Transactions on Information Theory, Vol. 8, No. 3, pp. 194195, 1962. https://doi.org/10.1109/TIT.1962.1057719.

18. F. Rusek, D. Persson, B. K. Lau, E. G. Larsson, T. L. Marzetta and F. Tufvesson, Scaling Up MIMO: Opportunities and Challenges with Very Large Arrays, Vol. 30, No. 1, pp. 40-60, 2013. https:// doi.org/10.1109/MSP.2011.2178495.
Publisher's Note Springer Nature remains neutral with regard to jurisdictional claims in published maps and institutional affiliations.

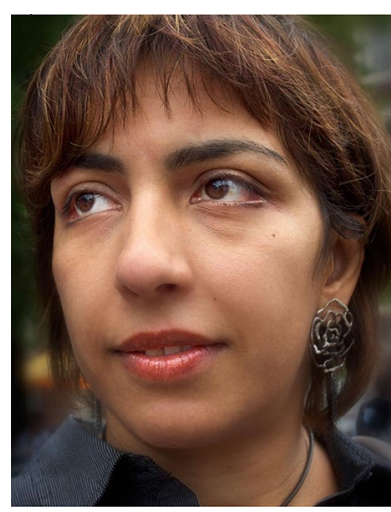

Golsa Ghiaasi received her Ph.D. in Electrical and Computer Engineering from The Ohio State University, USA in 2010. In 2014 she joined the Institute of Telecommunication at TUWien, Austria as a researcher with focus on inlab performance evaluation of $5 \mathrm{G}$ communication, in particular vehicular connectivity. She joined NTNU as an ERCIM fellow in 2016, where she is currently a researcher working on the field of empirical test and evaluation of $5 \mathrm{G}$ and beyond communication systems.

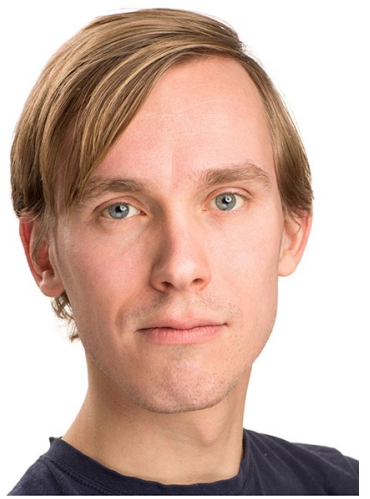

Jens Abraham received the Dipl.Ing. degree in Electrical Engineering from TU Dresden, Germany, in 2015. From 2015 to 2017 he was research assistant at the Astrophysics Group, Cavendish Laboratory, University of Cambridge, United Kingdom. He is currently a Ph.D. fellow with the Departement of Electronics at the Norwegian University of Science and Technology (NTNU) in Trondheim, Norway. His current research interests include measurements and modelling of massive MIMO radio channels.

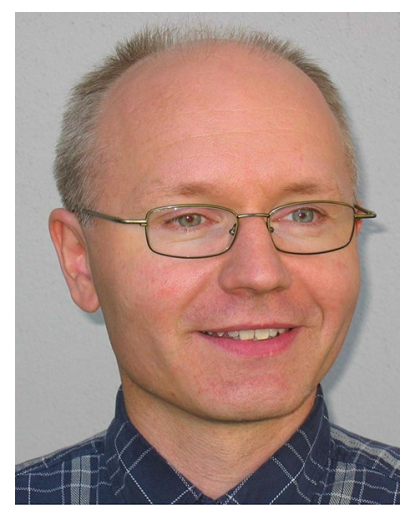

Egil Eide received his M.Sc. degree in Electronics Engineering from Norwegian Institute of Technology (NTH) in 1991 and his $\mathrm{PhD}$ degree in 2000 form Norwegian University of Science and Technology (NTNU). Dr. Eide's research interest is within the fields of antennas, radio systems, radar and remote sensing. In 2001 he formed the company 3d-Radar who manufactures high-resolution Ground Penetrating Radar systems based on Step-Frequency radar technology. Dr. Eide has since 2015 been Associate Professor in Radio Systems at NTNU. 


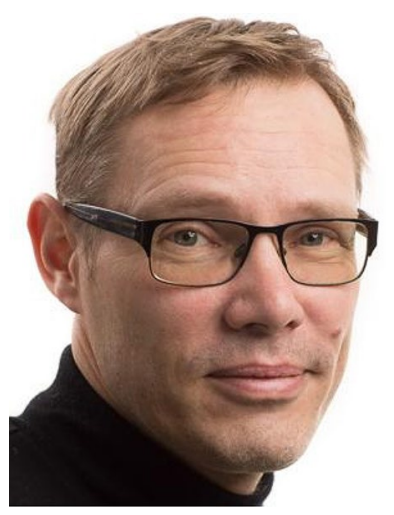

Torbjörn Ekman was born in Västerås, Sweden, in 1969. He received the M.Sc. degree in engineering physics in 1994 and the Ph.D. degree in signal processing in 2002, both from Uppsala University, Sweden. In 2006 he joined the Department of Electronics and Telecommunications at the Norwegian University of Science and Technology (NTNU) in Trondheim, Norway, where he now is a Professor. From 1997 to 1998 he was a visiting scientist at the Institute of Communications and Radio-

Frequency Engineering, Vienna University of Technology, Vienna, Austria, on a Marie Curie Grant. From 1999 to 2002, he was visiting

the Digital Signal Processing Group, University of Oslo, Norway. In 2002-2005, he made his postdoctoral studies at UniK, University Graduate Center, Kjeller, Norway. His current research interests include signal processing in wireless communications, massive MIMO, scheduling of radio resources, and dynamic modeling, and prediction of radio channels. He is currently participating in projects on a massive MIMO testbed, maritime and Arctic communication. 